\title{
Propuesta Pedagógica de Intervención en el Aula para Disminuir la Agresividad del Estudiante de Educación Secundaria en el I.E. Julio Cesar Tello - Hualmay 2015
}

\author{
Pedagogical Proposal Classroom Intervention to Reduce the Aggressiveness \\ of Secondary School Student in I.E. Julio Cesar Tello - Hualmay 2015
}

Juan Ernesto Ramos Manrique1, Reynado Francisco Cherrepano Manrique ${ }^{1}$, Delia Violeta Villafuerte Castro1, Yaneth Marlube Rivera Minaya', Julia Marina Bravo Montoya', Regulo Conde Curiñaupa', José Leonel Nicho Alcántara ${ }^{1}$

\section{RESUMEN}

Objetivo: Diseñar una propuesta pedagógica para disminuir la agresividad del estudiante de educación secundaria en la I.E Julio Cesar Tello- Hualmay 2015. Materiales y métodos: El diseño de la investigación fue cuasi experimental con pre prueba y post prueba con un grupo de comparación. La población estuvo conformada por 45 estudiantes de educación secundaria. Los instrumentos fueron la prueba de confiabilidad, la prueba de entrada, las sesiones de clase y la prueba de salida. Resultados: Finalmente se comprobó que la propuesta pedagógica de intervención en el aula disminuye la agresividad del estudiante de educación secundaria en la I.E Julio Cesar Tello-Hualmay 2015, debido a la significancia de la prueba Test U de MannWhitney en el post test aceptándose la hipótesis general y rechazándose la hipótesis nula.

Palabras clave: Propuesta, agresividad, intervención, pedagogía

\begin{abstract}
Objective: To design a pedagogical proposal to reduce the aggressiveness of secondary school student in I.E Julio Cesar Tello Hualmay 2015. Materials and Methods: The research design was quasi experimental with pretest and post-test with a comparison group. The population consisted of 45 high school students. The instruments were reliability test, test input, class sessions and output test. Results: Finally it was found that the pedagogical proposal of intervention in the classroom decreases aggressiveness high school student in IE Julio C. TelloHualmay 2015, due to the significance of the test Test Mann-Whitney $U$ in the post test accepting the general hypothesis and rejecting the null hypothesis.
\end{abstract}

Keywords: Proposal, aggression, intervention, pedagogy

${ }^{1}$ Facultad de Educación. Universidad Nacional José Faustino Sánchez Carrión. Lima - Perú Email: reconde@unifsc.edu.pe 


\section{INTRODUCCIÓN}

La agresividad estudiantil en una institución se presenta en todos los niveles de la educación a nivel nacional. En realidad se trata de un proceso para realizar las tareas básicas en una institución sistemáticamente. Un proceso como la gestión y administración, es mucho más fácil de comprender en partes, identificando aquellos problemas básicos; por tal razón la propuesta pedagógica permite que el desempeño docente sea más adecuado y un trabajo en equipo.

El comportamiento agresivo de los seres humanos es un tema estudiado desde siempre debido a las enormes implicancias que presenta para la vida en común y los altos costos que significa para la sociedad en términos de daño material, físico y emocional que causa. Este comportamiento, que tiene tanto determinantes biológicos como ambientales (Bandura, 1986; Bandura, Ross \& Ross, 1961; Raine, 2002), implica una intencionalidad de producir daño a otros, puede estar motivado por la cólera, el dolor, la frustración o el miedo, y algunas veces se manifiesta ya desde la primera infancia.

En las investigaciones realizadas; Chapa, S. (2012) Tarapoto Presencia de Violencia Familiar y su Relación con el nivel de Autoestima y Rendimiento Académico en Estudiantes de secundaria de la I.E N ${ }^{\circ}$ 0031 María Ulises Dávila Pinedo:

Queda demostrado que la violencia familiar está presente en la mayoría de los estudiantes del 1er y 2do de secundaria de la I.E Nº 0031 María Ulises Dávila Pinedo, con un porcentaje de $63 \%$ de la población estudiada.

- Con respecto al nivel de autoestima se concluye en el presente estudio que la mayoría de los estudiantes (64.5\%) no alcanzan un óptimo nivel de autoestima. Pues el $50.4 \%$ de estos tiene un nivel de autoestima Media, y el $14.1 \%$ de los alumnos tienen autoestima Baja, lo que significa que del $100 \%$ sólo el $35.6 \%$ tiene autoestima elevada.

- Con respecto al nivel de rendimiento académico se concluye que la mayoría de los estudiantes $(91.1 \%)$ no alcanzan un óptimo nivel de rendimiento.

- De estos alumnos el $30.4 \%$ tienen nivel medio y el $61.5 \%$ con nivel entre bajo y deficiente. Se demostró que si existe relación entre las variables nivel de autoestima y rendimiento académico con la variable violencia familiar, esto mediante la prueba de chi cuadrado, con un nivel de confianza de $95 \%$.

Por esta razón se consideró llevar a cabo la presente investigación, con el afán de determinar hasta qué punto resulta importante diseñar una propuesta pedagógica en base a la agresividad estudiantil, y comprender las características del problema para poder plantear sugerencias pertinentes para una mejor educación en el ámbito local y el desempeño docente, tan importante en el presente. Por tal motivo, nos hemos planteado como objetivo general Diseñar una Propuesta pedagógica de intervención en el aula, en la agresividad del estudiante de educación secundaria en la I.E Julio Cesar Tello-Hualmay 2015

La hipótesis planteada fue que la Propuesta pedagógica de intervención en el aula disminuye la agresividad del estudiante de educación secundaria en la I.E Julio Cesar Tello-Hualmay 2015, debido a la significancia de la prueba Test U de Mann- Whitney en el post test aceptándose la hipótesis general y rechazándose la hipótesis nula.

\section{MATERIALES Y MÉTODOS}

La presente investigación la Propuesta pedagógica de intervención en el aula para disminuir la agresividad del estudiante de educación secundaria en la I.E Julio Cesar Tello-Hualmay 2015. Pretende mostrar lineamientos metodológicos de tipo CuasiExperimental con pre-prueba, post-prueba con grupo de control y grupo experimental.

La variable dependiente estudiada fue Propuesta pedagógica y la variable independiente Agresividad de los estudiantes de Educación Secundaria.Se utilizó la prueba de normalidad muestra que los datos en las variables y dimensiones por grupos de control y experimental en el pre test y post test, no se distribuyen según la Ley Normal, ya que la "p" asociada a los contrastes de KolmogorovSmirnov da por debajo del nivel de significación alfa prefijado $(0,05)$.En este caso, nos obligó a tomar pruebas no paramétricas como la Prueba Test $U$ de MannWhitney. La muestra fue conformada por los estudiantes de educación secundaria, turno mañana un total de 300 alumnos de la Institución Educativa Julio Cesar Tello del distrito de Hualmay Provincia Huaura 2015. El tamaño de muestra es de 45 alumnos: 23 del grupo control y 22 del grupo experimental en dos aulas. Muestra no probabilística aleatoria estratificada.

\section{RESULTADOS}

La Propuesta pedagógica de intervención en el aula disminuye la agresividad del estudiante de educación secundaria en la I.E Julio Cesar Tello-Hualmay 2015, debido a la significancia de la prueba Test U de MannWhitney en el post test aceptándose la hipótesis general y rechazándose la hipótesis nula.

La Propuesta pedagógica de intervención en el aula disminuye la agresividad física del estudiante de educación secundaria en la I.E Julio Cesar TelloHualmay 2015, debido a la significancia de la prueba Test U de Mann- Whitney en el post test aceptándose la hipótesis general y rechazándose la hipótesis nula. 
La Propuesta pedagógica de intervención en el aula disminuye la agresividad verbal del estudiante de educación secundaria en la I.E Julio Cesar TelloHualmay 2015, debido a la significancia de la prueba Test $U$ de Mann- Whitney en el post test aceptándose la hipótesis general y rechazándose la hipótesis nula.

La Propuesta pedagógica de intervención en el aula disminuye la agresividad psicológica del estudiante de educación secundaria en la I.E Julio Cesar TelloHualmay 2015, debido a la significancia de la prueba Test $U$ de Mann- Whitney en el post test aceptándose la hipótesis general y rechazándose la hipótesis nula.

La Propuesta pedagógica de intervención en el aula disminuye la agresividad social del estudiante de educación secundaria en la I.E Julio Cesar TelloHualmay 2015, debido a la significancia de la prueba Test $U$ de Mann- Whitney en el post test aceptándose la hipótesis general y rechazándose la hipótesis nula.

Tabla 1: Pruebas de normalidad

\begin{tabular}{|c|c|c|c|}
\hline \multirow{2}{*}{ Variables y Dimensiones } & \multicolumn{3}{|c|}{ Kolmogorov-Smirnov $^{a}$} \\
\hline & Estadistico & GI & Sig. \\
\hline e test niv & 183 & 45 & 001 \\
\hline st text ni & 226 & 45 & ,000 \\
\hline Pre test nivel alcanzado de agresividad física & ,385 & 45 & ,000 \\
\hline Pres test nivel alcanzado de agresividad verbal & 214 & 45 & ,000 \\
\hline Pre test nivel alcanzado de agresividad psicológica & ,281 & & \\
\hline 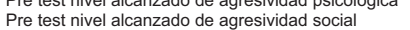 & 211 & 45 & 000 \\
\hline 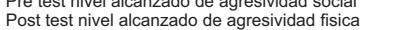 & 182 & 45 & 001 \\
\hline Post test nivel alcanzado de agresividad verbal & 210 & 45 & ,000 \\
\hline Post te & 267 & 45 & ,000 \\
\hline Post test niv & 170 & 45 & ,002 \\
\hline
\end{tabular}

Tabla 2: La agresividad del estudiante de educación secundaria en la I.E. Julio Cesar Tello - Hualmay 2015

\begin{tabular}{|c|c|c|c|}
\hline \multirow{2}{*}{ Estadistico } & \multicolumn{2}{|c|}{ Grupo } & \multirow{2}{*}{$\begin{array}{c}\text { Test U de } \\
\text { Mann-Whitney }\end{array}$} \\
\hline & Control $(n=23)$ & Experimental $(n=22)$ & \\
\hline $\begin{array}{l}\text { Media } \\
\text { Desv. tip. }\end{array}$ & $\begin{array}{l}5.10 \\
1.44\end{array}$ & $\begin{array}{ll}\text { Pretest } & 5.49 \\
& 1.54 \\
& \end{array}$ & $\begin{array}{l}\mathrm{U}=186.500 \\
\mathrm{Z}=-0.380 \\
p=0.704\end{array}$ \\
\hline $\begin{array}{l}\text { Media } \\
\text { Desv. tip. }\end{array}$ & $\begin{array}{r}12.55 \\
1.36\end{array}$ & $\begin{array}{r}\text { Post test } \\
6.55 \\
1.90\end{array}$ & $\begin{array}{ll}\mathrm{U}= & 6.00 \\
\mathrm{Z}= & -5.308 \\
p= & 0.000\end{array}$ \\
\hline
\end{tabular}

Tabla 3: La agresividad física del estudiante de educación secundaria en la I.E Julio Cesar TelloHualmay 2015.

\begin{tabular}{|c|c|c|c|}
\hline \multirow{2}{*}{ Estadistico } & \multicolumn{2}{|c|}{ Grupo } & \multirow{2}{*}{$\begin{array}{c}\text { Test U de } \\
\text { Mann-Whitney }\end{array}$} \\
\hline & Control $(n=23)$ & Experimental $(n=22)$ & \\
\hline Media & 1.15 & $\begin{array}{ll}\text { Pretest } & 1.25\end{array}$ & $U=181.000$ \\
\hline Desv. tip. & 1.09 & 1.08 & $\begin{array}{l}Z=-0.566 \\
p=0.571\end{array}$ \\
\hline Media & 3.00 & Post test 1.05 & \\
\hline Desv. tip. & 0.99 & 0.87 & $\begin{array}{ll}Z= & -4.129 \\
p= & 0.000\end{array}$ \\
\hline
\end{tabular}

Tabla 4: La agresividad verbal del estudiante de educación secundaria en la I.E Julio Cesar TelloHualmay 2015.

\begin{tabular}{|c|c|c|c|}
\hline \multirow{2}{*}{ Estadistico } & \multicolumn{2}{|c|}{ Grupo } & \multirow{2}{*}{$\begin{array}{c}\text { Test U de } \\
\text { Mann-Whitney }\end{array}$} \\
\hline & Control (n=23) & Experimental $(n=22)$ & \\
\hline Media & 2.00 & Pretest 2.20 & $U=163.000$ \\
\hline Desv. tip. & 0.89 & 1.07 & $\begin{array}{lr}Z= & -1.057 \\
p= & 0.291\end{array}$ \\
\hline Media & 4.05 & Post test 2.00 & $U=47000$ \\
\hline Desv. tip. & 1.04 & 0.94 & $\begin{array}{ll}Z= & -4.250 \\
p= & 0.000\end{array}$ \\
\hline
\end{tabular}

Tabla 5: La agresividad psicológica del estudiante de educación secundaria en la I.E Julio Cesar TelloHualmay 2015.

\begin{tabular}{|c|c|c|c|}
\hline \multirow{2}{*}{ Estadistico } & \multicolumn{2}{|c|}{ Grupo } & \multirow{2}{*}{$\begin{array}{c}\text { Test U de } \\
\text { Mann-Whitney }\end{array}$} \\
\hline & Control (n=23) & Experimental $(n=22)$ & \\
\hline Media & 1.00 & Pretest 1.16 & $U=172.000$ \\
\hline Desv. tip. & 0.93 & 1.04 & $\begin{array}{l}Z=-0.801 \\
p=0.423\end{array}$ \\
\hline $\begin{array}{l}\text { Media } \\
\text { Desv. tip. }\end{array}$ & $\begin{array}{l}2.00 \\
1.03\end{array}$ & $\begin{aligned} \text { Post test } & 1.00 \\
& 0.60\end{aligned}$ & $\begin{array}{l}\mathrm{U}=26.00 \\
\mathrm{Z}=-4.864 \\
p=0.000\end{array}$ \\
\hline
\end{tabular}

Tabla 6: La agresividad social del estudiante de educación secundaria en la I.E Julio Cesar TelloHualmay 2015.

\begin{tabular}{|c|c|c|c|}
\hline \multirow{2}{*}{ Estadistico } & \multicolumn{2}{|c|}{ Grupo } & \multirow{2}{*}{$\begin{array}{c}\text { Test U de } \\
\text { Mann-Whitney }\end{array}$} \\
\hline & Control (n=23) & Experimental $(n=22)$ & \\
\hline $\begin{array}{l}\text { Media } \\
\text { Desv. tip. }\end{array}$ & $\begin{array}{l}2.00 \\
0.59\end{array}$ & $\begin{array}{ll}\text { Pretest } & 2.14 \\
& 0.63\end{array}$ & $\begin{array}{l}\mathrm{U}=175.500 \\
\mathrm{Z}=\quad-0.745 \\
p=0.456\end{array}$ \\
\hline $\begin{array}{l}\text { Media } \\
\text { Desv. tip. }\end{array}$ & $\begin{array}{l}4.20 \\
0.59\end{array}$ & $\begin{array}{r}\text { Post test } \\
3.00 \\
0.41\end{array}$ & $\begin{aligned} \mathrm{U} & =32.00 \\
\mathrm{Z} & =-4.873 \\
p= & 0.000\end{aligned}$ \\
\hline
\end{tabular}

\section{DISCUSIÓN}

En el pre test La agresividad del estudiante de educación secundaria en la I.E Julio Cesar TelloHualmay 2015 con un $95 \%$ de confiabilidad y un nivel de significancia del $5 \%$ de acuerdo a la prueba no paramétrica $U$ de Mann-Whitney para muestras independientes, tanto para el grupo de control y experimental, presentan condiciones iniciales similares (U-Mann-Whitney $p=0.704$ ), ya que sobrepasa el valor de significancia.

En el post test de la misma forma, el desarrollo psicomotor presentan condiciones diferentes de acuerdo a la prueba no paramétrica $U$ de MannWhitney, $(p=0.000)$ tanto para el grupo de control y experimental, por lo que, los estudiantes del grupo experimental obtuvieron mejores resultados (Promedio $=6.55)$ después de la aplicación de la propuesta pedagógica de intervención en el aula, respecto a los estudiantes del grupo de control $($ Promedio $=12.5)$, disminuyendo significativamente la agresividad del estudiante.

Se puede afirmar que existe evidencia para concluir que la Propuesta pedagógica de intervención en el aula disminuye la agresividad del estudiante de educación secundaria en la I.E Julio Cesar TelloHualmay 2015, debido a la significancia de la prueba Test $U$ de Mann- Whitney en el post test aceptándose la hipótesis general y rechazándose la hipótesis nula. Como se muestra en la tabla 2.

En el pre test La agresividad física del estudiante de educación secundaria en la I.E Julio Cesar TelloHualmay 2015 con un 95\% de confiabilidad y un nivel de significancia del $5 \%$ de acuerdo a la prueba no paramétrica $U$ de Mann-Whitney para muestras independientes, tanto para el grupo de control y 
experimental, presentan condiciones iniciales similares (U-Mann-Whitney $p=0.571$ ), ya que sobrepasa el valor de significancia.

En el post test de la misma forma, el desarrollo psicomotor presentan condiciones diferentes de acuerdo a la prueba no paramétrica $U$ de MannWhitney, $(p=0.000)$ tanto para el grupo de control y experimental, por lo que, los estudiantes del grupo experimental obtuvieron mejores resultados (Promedio $=1.05)$ después de la aplicación de la propuesta pedagógica de intervención en el aula, respecto a los estudiantes del grupo de control (Promedio $=3.00)$, disminuyendo significativamente la agresividad física del estudiante.

Se puede afirmar que existe evidencia para concluir que la Propuesta pedagógica de intervención en el aula disminuye la agresividad física del estudiante de educación secundaria en la I.E Julio Cesar TelloHualmay 2015, debido a la significancia de la prueba Test U de Mann- Whitney en el post test aceptándose la hipótesis general y rechazándose la hipótesis nula.Como se muestra en la tabla 3.

En el pre test La agresividad verbal del estudiante de educación secundaria en la I.E Julio Cesar TelloHualmay 2015 con un $95 \%$ de confiabilidad y un nivel de significancia del $5 \%$ de acuerdo a la prueba no paramétrica $U$ de Mann-Whitney para muestras independientes, tanto para el grupo de control y experimental, presentan condiciones iniciales similares (U-Mann-Whitney $p=0.291$ ), ya que sobrepasa el valor de significancia.

En el post test de la misma forma, el desarrollo psicomotor presentan condiciones diferentes de acuerdo a la prueba no paramétrica $U$ de MannWhitney, $(p=0.000)$ tanto para el grupo de control y experimental, por lo que, los estudiantes del grupo experimental obtuvieron mejores resultados (Promedio $=2.00$ ) después de la aplicación de la propuesta pedagógica de intervención en el aula, respecto a los estudiantes del grupo de control (Promedio $=4.05)$, disminuyendo significativamente la agresividad verbal del estudiante.

Se puede afirmar que existe evidencia para concluir que la Propuesta pedagógica de intervención en el aula disminuye la agresividad verbal del estudiante de educación secundaria en la I.E Julio Cesar TelloHualmay 2015, debido a la significancia de la prueba Test U de Mann- Whitney en el post test aceptándose la hipótesis general y rechazándose la hipótesis nula.Como se muestra en la tabla 4.
En el pre test la agresividad psicológica del estudiante de educación secundaria en la I.E Julio Cesar Tello-Hualmay 2015 con un $95 \%$ de confiabilidad y un nivel de significancia del $5 \%$ de acuerdo a la prueba no paramétrica $U$ de MannWhitney para muestras independientes, tanto para el grupo de control y experimental, presentan condiciones iniciales similares (U-Mann-Whitney $p=0.423$ ), ya que sobrepasa el valor de significancia.

En el post test de la misma forma, el desarrollo psicomotor presentan condiciones diferentes de acuerdo a la prueba no paramétrica $U$ de MannWhitney, $(p=0.000)$ tanto para el grupo de control y experimental, por lo que, los estudiantes del grupo experimental obtuvieron mejores resultados (Promedio $=1.00)$ después de la aplicación de la propuesta pedagógica de intervención en el aula, respecto a los estudiantes del grupo de control (Promedio $=2.00)$, disminuyendo significativamente la agresividad psicológica del estudiante.

Se puede afirmar que existe evidencia para concluir que la Propuesta pedagógica de intervención en el aula disminuye la agresividad psicológica del estudiante de educación secundaria en la I.E Julio Cesar Tello-Hualmay 2015, debido a la significancia de la prueba Test U de Mann- Whitney en el post test aceptándose la hipótesis general y rechazándose la hipótesis nula.Como se muestra en la tabla 5.

En el pre test la agresividad social del estudiante de educación secundaria en la I.E Julio Cesar TelloHualmay 2015 con un $95 \%$ de confiabilidad y un nivel de significancia del $5 \%$ de acuerdo a la prueba no paramétrica $U$ de Mann-Whitney para muestras independientes, tanto para el grupo de control y experimental, presentan condiciones iniciales similares (U-Mann-Whitney $p=0.456)$, ya que sobrepasa el valor de significancia.

En el post test de la misma forma, el desarrollo psicomotor presentan condiciones diferentes de acuerdo a la prueba no paramétrica $U$ de MannWhitney, $(p=0.000)$ tanto para el grupo de control y experimental, por lo que, los estudiantes del grupo experimental obtuvieron mejores resultados (Promedio $=3.00$ ) después de la aplicación de la propuesta pedagógica de intervención en el aula, respecto a los estudiantes del grupo de control (Promedio $=4.20)$, disminuyendo significativamente la agresividad social del estudiante.

Se puede afirmar que existe evidencia para concluir que la Propuesta pedagógica de intervención en el 
aula disminuye la agresividad social del estudiante de educación secundaria en la I.E Julio Cesar TelloHualmay 2015, debido a la significancia de la prueba Test U de Mann- Whitney en el post test aceptándose la hipótesis general y rechazándose la hipótesis nula.Como se muestra en la tabla 6.

\section{REFERENCIAS BIBLIOGRÁFICAS}

Jadue. (2003) Ensayos transformaciones familiares en chile: riesgo creciente para el desarrollo emocional, psicosocial y la educación de los hijos. Páginas 115-126

Proyecto de tesis universidad José Faustino Sánchez Carrión "la violencia familiar y su influencia en el rendimiento académico de los alumnos de 6to grado de primaria de la I.E Luis Fabio Xammar jurado-huacho-2011.

Barraga, (2012) Incidencia de la violencia intrafamiliar en el rendimiento académico de los estudiantes de la Escuela Fiscal Mixta Vespertina -Ecuador.

Corsi. (1995) Violencia Familiar. Una mirada interdisciplinaria sobre un grave problema social. Buenos Aires: Paidós. Argentina

Gómez, A. (2007) El ambiente familiar y el desarrollo socio emocional en los niños de 3 a 5 años del PRONOEI S. XII, en el Distrito de Gregorio Albarracín del departamento de Tacna, 2006.
Rojas, H. (2004). Habilidades sociales, Perú: Lunagraf E.I.R EDT. Revista Ciencias.

Chapa. (2012) Tarapoto Presencia de Violencia Familiar y su Relación con el Nivel de Autoestima y Rendimiento Académico en Estudiantes de Secundaria de la I.E n 0031 María Ulises Dávila pinedo. Recuperado el 03 de enero de 2014.

Jiménez, Toledo, (2013) Piura recuperado el 04 de enero del 2014.

Guadalupe, Tesis de la Maestría en Educación superior. La relación interpersonal entre el docente universitario y el estudiante. Recuperado el 23 de diciembre del 2013.

Machuca, (2010) Arequipa proyecto de investigación. Desarrollar las Habilidades sociales en los estudiantes del 1er. grado de educación secundaria de la I.E. granunidad escolar Miguel Grau Puacarpata Carlos Mariátegui. Recuperado el 03 de enero del 2014.

Palacios Andrea (2010) violencia familiar visitado el día 06 de diciembre del 2013.

Ramírez y Chero (1998). La Violencia familiar y su influencia en el desarrollo Psicomotor de los alumnos del $1^{\circ}$ grado de Primaria del C.E "Hermanos Meléndez "La Unión. Piura. Recuperado el 26 de mayo del 2012

Real academia.

Santamaría. (2010) Caracas Venezuela Violencia Familiar y Maltrato Infantil. 